\title{
A Case Study on the Investigation and Prediction of A1C Variances over Six Periods Using GH-Method: Math-Physical Medicine
}

\author{
Gerald C. Hsu*
}

Medical Research Scientist, EclaireMD Fondation, 7 Oak Haven Way, Woodside, CA 94062 USA.

*Corresponding Author: Gerald C. Hsu, Medical Research Scientist, EclaireMD Fondation, 7 Oak Haven Way, Woodside, CA 94062 USA.

\section{INTRODUCTION}

In this case study, the author analyzed, predicted, and interpreted a type 2 diabetes (T2D) patient's hemoglobin A1C variances based on six periods data utilizing the GHMethod: math-physical medicine approach.

\section{METHOD}

There are six hemoglobin A1C checkup results at the same hospital:

$6.7 \%$ on $4 / 9 / 2017$

$6.1 \%$ on $9 / 12 / 2017$

$6.9 \%$ on $1 / 26 / 2018$

$6.5 \%$ on $6 / 29 / 2018$

$6.6 \%$ on $10 / 22 / 2018$

$6.8 \%$ on $4 / 4 / 2019$

$6.5 \%$ on $9 / 25 / 2019$

The author selected six periods of almost equal length with about five months each and then observed their measured A1C changes (variances) as follows:

Period A (4/1/2017 - 8/31/2017): -0.6\%

Period B (9/1/2017 - 1/31/2018): +0.8\%
Period C (2/1/2018 - 6/30/2018): -0.4\%

Period D (6/29/2018 - 10/22/2018): +0.1\%

Period E (10/22/2018 - 4/4/2019): +0.2\%

Period F (4/4/2019 - 9/25/2019): -0.3\%

He applied his developed GH-Method: mathphysical medicine (MPM approach) to analyze the contribution of six A1C variances:

(1) A1C variances contributed by FPG

(2) FPG variance due to weight change

(3) Colder weather impact on FPG

(4) A1C variances contributed by PPG

(5) PPG variance due to carbs/sugar intake

(6) PPG variance due to post-meal walking

(7) Warm weather impact on PPG

\section{RESUlts}

Based on the author's numerous publications of HbA1C contributions by FPG and PPG, along with the prediction models of two glucoses and A1C, Figure 1 shows his FPG and PPG during the most recent period $\mathrm{F}$ and Table 1 displays a step-by-step calculation on how to derive and interpret the causes of the these $\mathrm{A} 1 \mathrm{C}$ variances.

Table 1: Calculation of AlC Variances (six periods)

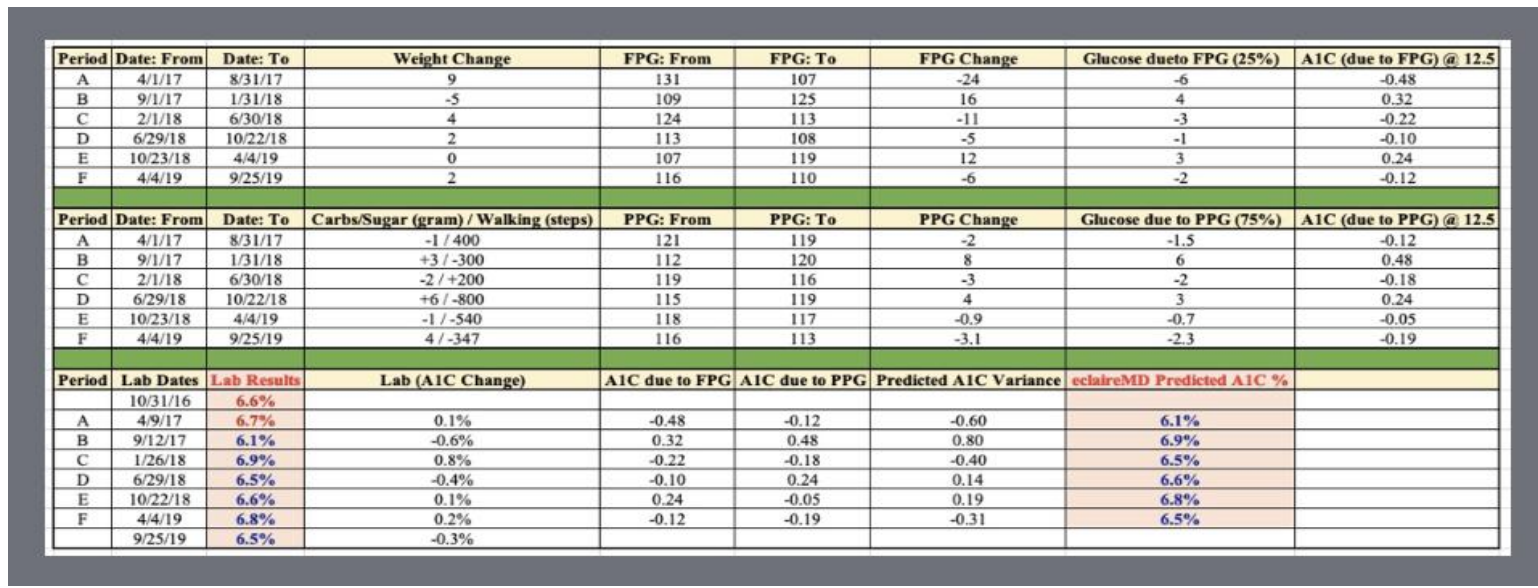


A Case Study on the Investigation and Prediction of A1C Variances over Six Periods Using GH-Method: Math-Physical Medicine

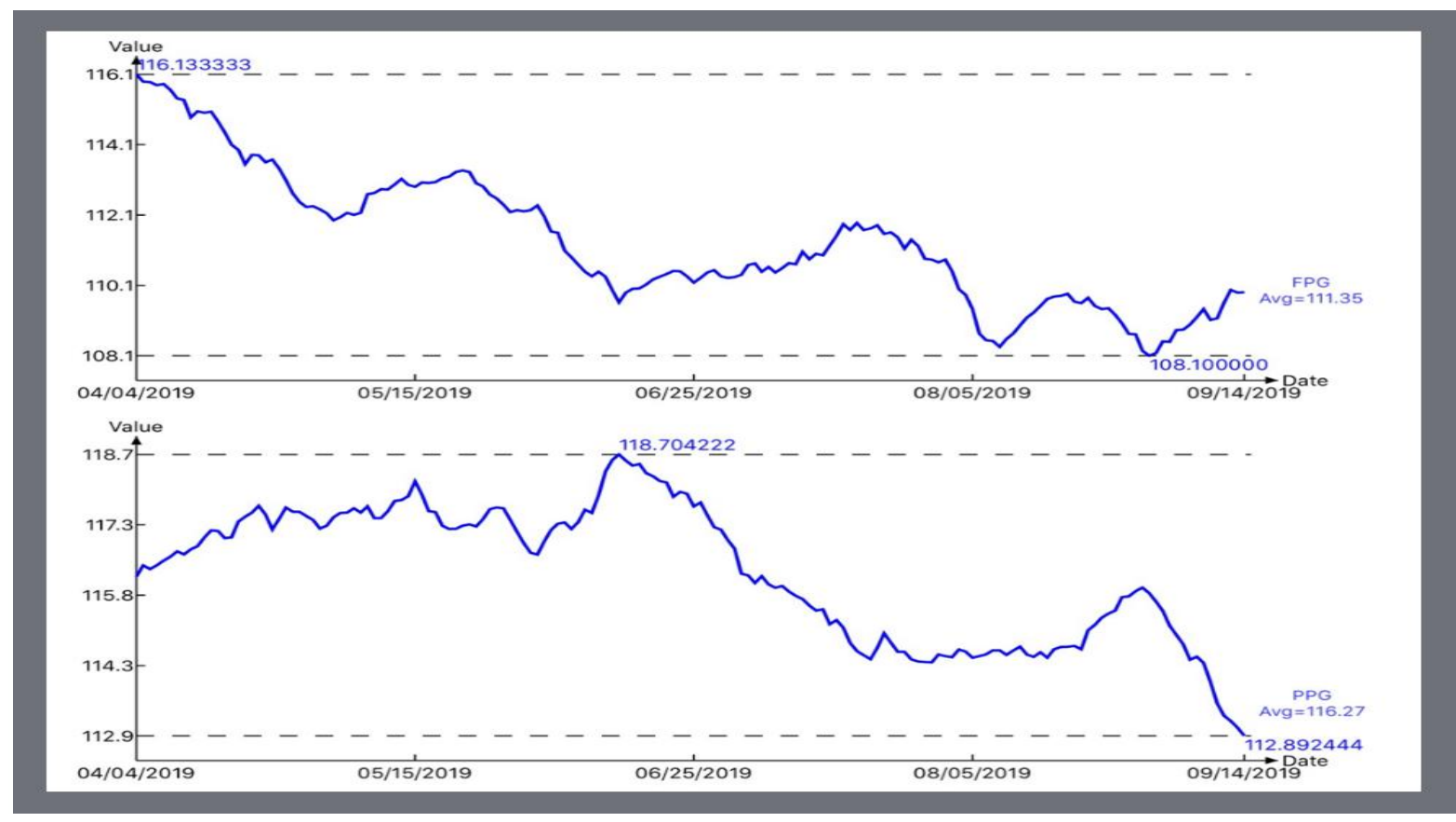

Figure 1: FPG and PPG (90-days moving average from 4/4/2019 to 9/25/2019)

As shown in Table 1, his predicted A1C variances completely match the test results from the laboratory.

\section{CONCLUSION}

The A1C case study focused on five periods within 908 days. It contains 2,724 meals data, including key contribution factors such as carbs/sugar intake, post-meal exercise, weather, etc. This study has demonstrated a high degree of accuracy on calculating and predicting the patient's forthcoming A1C value by using the GH-Method: math-physical medicine (MPM) approach. Once the healthcare professionals and T2D patients understand and learn this skill for the HbA1C prediction method, the patient's overall T2D condition can then be more easily under control. The purpose of this research paper is to help T2D patients to prevent further damage to their internal organs caused by high $\mathrm{HbA} 1 \mathrm{C}$, while waiting for the laboratory test results.

\section{REFERENCES}

[1] Hsu, Gerald C. (2018). Using Math-Physical Medicine to Control T2D via Metabolism
Monitoring and Glucose Predictions. Journal of Endocrinology and Diabetes, 1(1), 1-6. Retrieved from http://www. kosmos publishers. com/wp-content/uploads/ 2018/06/JEAD-1011.pdf

[2] Hsu, Gerald C. (2018, June). Using MathPhysical Medicine to Analyze Metabolism and Improve Health Conditions. Video presented at the meeting of the 3rd International Conference on Endocrinology and Metabolic Syndrome 2018, Amsterdam, Netherlands.

[3] Hsu, Gerald C. (2018). Using Signal Processing Techniques to Predict PPG for T2D. International Journal of Diabetes \& Metabolic Disorders, 3(2),1-3. Retrieved from https:// www.opastonline.com/wp-content/ uploads/ 2018/06/using-signal-processing-techniques-topredict-ppg-for-t2d-ijdmd-18.pdf

[4] Hsu, Gerald C. (2018). Using Math-Physical Medicine and Artificial Intelligence Technology to Manage Lifestyle and Control Metabolic Conditions of T2D. International Journal of Diabetes \& Its Complications, 2(3), 1-7. Retrieved from http:/ /cmepub. com/pdfs/using-mathphysical-medicine-andartificial-intelligence-technology-to-managelifestyle-and-control-metabolic-conditions-oft2d-412.pdf

Citation: Gerald C. Hsu, "A Case Study on the Investigation and Prediction of AlC Variances over Six Periods Using GH-Method: Math-Physical Medicine”, International Journal of Research Studies in Medical and Health Sciences. 2020; 5(5): 36-37.

Copyright: (c) 2020 Gerald C. Hsu, This is an open-access article distributed under the terms of the Creative Commons Attribution License, which permits unrestricted use, distribution, and reproduction in any medium, provided the original author and source are credited. 